\title{
Laser Raman Spectroscopy with Different Excitation Sources and Extension to Surface Enhanced Raman Spectroscopy
}

\author{
Md. Wahadoszamen, Arifur Rahaman, Nabil Md. Rakinul Hoque, Aminul I Talukder, \\ Kazi Monowar Abedin, and A. F. M. Yusuf Haider \\ Department of Physics, University of Dhaka, Dhaka 1000, Bangladesh
}

Correspondence should be addressed to Md. Wahadoszamen; wahado@univdhaka.edu and Arifur Rahaman; phdu.arif@yahoo.com

Received 23 August 2014; Accepted 6 October 2014

Academic Editor: Nikša Krstulović

Copyright ( $) 2015 \mathrm{Md}$. Wahadoszamen et al. This is an open access article distributed under the Creative Commons Attribution License, which permits unrestricted use, distribution, and reproduction in any medium, provided the original work is properly cited.

A dispersive Raman spectrometer was used with three different excitation sources (Argon-ion, He-Ne, and Diode lasers operating at $514.5 \mathrm{~nm}, 633 \mathrm{~nm}$, and $782 \mathrm{~nm}$, resp.). The system was employed to a variety of Raman active compounds. Many of the compounds exhibit very strong fluorescence while being excited with a laser emitting at UV-VIS region, hereby imposing severe limitation to the detection efficiency of the particular Raman system. The Raman system with variable excitation laser sources provided us with a desired flexibility toward the suppression of unwanted fluorescence signal. With this Raman system, we could detect and specify the different vibrational modes of various hazardous organic compounds and some typical dyes (both fluorescent and nonfluorescent). We then compared those results with the ones reported in literature and found the deviation within the range of $\pm 2 \mathrm{~cm}^{-1}$, which indicates reasonable accuracy and usability of the Raman system. Then, the surface enhancement technique of Raman spectrum was employed to the present system. To this end, we used chemically prepared colloidal suspension of silver nanoparticles as substrate and Rhodamine $6 \mathrm{G}$ as probe. We could observe significant enhancement of Raman signal from Rhodamine $6 \mathrm{G}$ using the colloidal solution of silver nanoparticles the average magnitude of which is estimated to be $10^{3}$.

\section{Introduction}

Raman Spectroscopy is based on the Raman Effect, which is the inelastic scattering of photons by molecules or molecular aggregates [1]. In typical Laser Raman Spectroscopy (LRS), light from a laser in the UV-Vis-IR range of a known frequency and polarization is allowed to be scattered from a Raman active sample. The scattered light is then analyzed for frequency and polarization [2]. If the emitted photon has the same wavelength as the absorbing photon; the phenomenon is known as Rayleigh scattering [3]. A small fraction of the incident photons, about 1 in $10^{7}$, undergoes interaction with the targeted sample and thereby experiences inelastic scattering [4]. In such a case, the incident laser light interacts with molecular vibrations, phonons, or other possible excitation sources present in the system and thereby resulting in a shift in the energy of the scattered laser photons either up or down. If the frequency of the scattered photon is lowered, then the phenomenon is called Stokes scattering. If the energy of the photon is shifted upward it is called anti-Stokes scattering. The shift in energy provides vital information about the inherent vibrational modes of the molecule or a molecular aggregate under investigation. Although, Raman scattered light is frequency-shifted with respect to the excitation frequency, the magnitude of the shift in energy is independent of the excitation frequency [5]. The resulting "Raman shift" is therefore an intrinsic property of a molecule, which makes the Raman Spectroscopy a powerful experimental tool for analytical studies. In fact, Raman spectroscopy is commonly designed to monitor intrinsic vibrational spectral fingerprints of a molecule and/or molecular composite. Therefore, Raman spectroscopy can primarily be used to diagnose the presence of a molecule and/or molecular composite in an ensemble [6]. It is also capable of giving detailed information about molecular structure and quantitative analysis [7].

Raman signal arises mainly from a magnetic dipole active vibrational transition of a molecule and thus intrinsically very week in nature. Therefore, it is sometimes very difficult 
to detect the Raman signal of the compounds that are highly fluorescent, as the very weak Raman signals of such compounds are often severely masked by the intense fluorescence signal. As a matter of fact, sensitivity remained a vital issue for Raman spectroscopy which resulted in Raman spectroscopy not being widely used earlier. Therefore, huge flurry of research on Raman spectroscopy and its intensity optimization are found over recent few decades [8]. The simple approach to avoid the fluorescence emission can be to select the suitable laser excitation wavelength. The choice of a near infrared (NIR) excitation wavelength can avoid strong fluorescence, since, in the NIR excitation, the photon does not have enough energy to induce molecular fluorescence. The combination of metal surface (nano) technology and Raman spectroscopy has given rise to "Surface Enhanced Raman Spectroscopy" (SERS) $[9,10]$. SERS has so far eased many difficulties associated with the intensity optimization of intrinsically weak Raman signal(s). SERS is a surface sensitive technique that results in the enhancement of Raman scattering by adsorbing molecules on rough metal surfaces or on the surface of the colloidal nano-clusters of suitable dimension. The enhancement factor can be as much as $10^{4}-10^{5}$, which allows the technique to be sensitive enough to detect single molecule [11]. In this paper, we used a homebuilt Laser Raman system with three different excitation sources and applied the Raman system to a variety of Raman active compounds (hazardous organic compounds and some typical dyes). Many of the selected compounds have very strong intrinsic fluorescence. Therefore, the construction of Raman system with variable and suitable excitation laser sources was primarily aimed to provide a desired flexibility toward the suppression of unwanted fluorescence while detecting the Raman signal. Then, an effort is made to evaluate the potential of Raman spectroscopy to achieve SERS activity. To this end, we used chemically prepared colloidal suspension of silver nanoparticles $(\mathrm{AgN})$ as substrate and Rhodamine $6 \mathrm{G}(\mathrm{R} 6 \mathrm{G})$ as probe.

\section{Experimental}

We previously constructed a dispersive laser Raman system with $\mathrm{He}-\mathrm{Ne}$ or diode laser as excitation sources and used it to acquire and measure the Raman spectra of a variety of molecules, pharmaceuticals, and edible oils [13-15]. In this experiment, we modified the Raman system to operate with three types of excitation lasers, Argon-Ion laser, HeNe laser, and Diode laser operating at $514.5 \mathrm{~nm}, 633 \mathrm{~nm}$, and $782 \mathrm{~nm}$, respectively. Out of the three excitation sources, we have used mostly the Argon-Ion laser and Diode laser for our experiments. The schematic layout of the modified Laser Raman system operated with Argon-Ion laser is shown in the Figure 1.

Argon-Ion laser is a broadband laser which emits simultaneously at a number of different wavelengths (e.g., $454.6 \mathrm{~nm}$, $488.0 \mathrm{~nm}, 496.5 \mathrm{~nm}$, and $514.5 \mathrm{~nm}$ ) at the head [16]. Among the different wavelengths, we used the strongest and welldistinguished $514.5 \mathrm{~nm}$ one as the excitation wavelength for the Raman system. The extraction of the $514.5 \mathrm{~nm}$ wavelength was done as follows: the broadband output of the Argon-Ion laser was dispersed by a prism, then the $514.5 \mathrm{~nm}$ emission was separated upon passing the well separated light through a narrow homemade pinhole of suitable dimension (diameter around $5 \mathrm{~mm}$ ).

The beam was then focused on the cylindrical glass vial containing sample and the Raman-scattered radiations were collected at an oblique geometry at $(45-90)^{\circ}$ by a largeaperture convex lens (f/0.98) which was ultimately focused to the entrance window of the optical fiber. A suitable holographic notch filter was used just in the front of the entrance of the optical fiber, which in turn allows the desired Raman signals to pass through it and thus attenuating the unwanted Rayleigh scattering. The entire system was used in a dark room to avoid densitization of the sensitive detection system by ambient light. Furthermore, a light tight housing (with beam dump in the path way of the laser beam) was used to cover the sample, lenses, filter, mirror, and entrance slit of the spectrograph in order to prevent light reaching from computer monitor and other glowing/radiant objects. The outlet of the optical fiber was connected to the entrance slit of the spectrograph. Inside the spectrograph, the frequency-shifted signal was dispersed by the triple grating turret to resolve spectrally the available Raman signatures. The spectrometer used is an Acton SpectraPro-2758 system with a CzernyTurner configuration having a focal length of $750 \mathrm{~mm}$. It has three ruled gratings $(150$ lines $/ \mathrm{mm}$ blazed at $500 \mathrm{~nm}$, 300 lines $/ \mathrm{mm}$ blazed at $500 \mathrm{~nm}$, and 300 lines $/ \mathrm{mm}$ blazed at $1 \mu \mathrm{m})$, which can be selected at will electronically. The output port of the spectrometer is equipped with an ultrasensitive CCD camera (Princeton Instruments PIXIS100B), which records the spectrum. The camera has a $1340 \times 100$ pixels imaging array and was cooled to $-65^{\circ} \mathrm{C}$ by a Peltier cooler in order to reduce thermal noise. Both the CCD camera and the spectrometer were controlled by computer using the software Winspec/32. Throughout the experiment, the instrumental parameters were as follows unless stated otherwise in the figure caption: focused mode (Readout mode: Full Frame, Digitization rate: $100 \mathrm{kHz}$, slow type), $100 \%$ laser, entrance slit width 30 microns, $1340 \times 100 \mathrm{CCD}$ array $\left(-65^{\circ} \mathrm{C}\right.$ detector temperature), grating selection of 300 lines $/ \mathrm{mm}$ grooves blazed at $500 \mathrm{~nm}$ for Argon-Ion/He-Ne laser excitation, and 300 lines $/ \mathrm{mm}$ grooves blazed at $1 \mu \mathrm{m}$ for the diode laser excitation. The collection time for spectral acquisition with good signal-to-noise ratio depended on Raman scattering intensity; however, we used 60 seconds acquisition time quite frequently. The Raman Spectrometer reported herein possesses multitude of advantages compared to some commercially available portable Raman spectrometers. Some merits, among the others, of our Raman spectrometer can be summed as follows. (1) It provides one with the ultimate flexibility towards choosing the excitation wavelength. The spectrometer is designed on top of a large optical table with three laser excitation sources in such way that, within the spectrometer configuration, one can easily change (based on the necessity) the source of the excitation during the course of experiments. (2) The sample irradiation and the Raman signal collection optics of this spectrometer are assembled on top of a fairly spacious mechanically movable lab-jack, thereby enabling one to change the irradiation as well as the collection geometry at will for the quest of better 


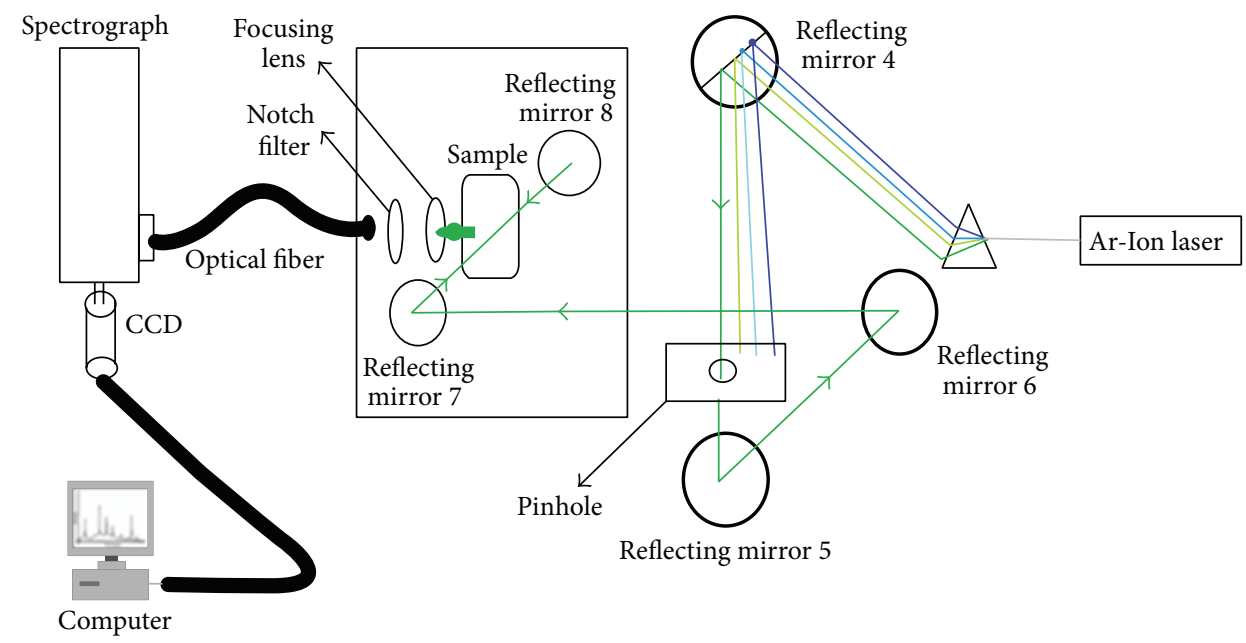

FIGURE 1: Schematic diagram of the modified Raman System using Argon-Ion laser (514.5 nm emission).

Raman signal. This novel design facilitated for us to inspect characteristics lines as close as $200 \mathrm{~cm}^{-1}$ from the laser line with a resolution less than $2 \mathrm{~cm}^{-1}$ ample for analyzing Raman fingerprints for many compounds. (3) The Raman signal is collected through an optical fiber and thus, once the sample collection geometry is changed to some extent for optimizing the Raman signal, one can easily change the position of the head of the optical fiber to make it compatible with the new geometry. (4) The detection system of this spectrometer is equipped with highly sensitive spectrograph, Acton SpectraPro-2758. The frequency selector of this spectrograph has high-aperture optics and feature coma-corrected CzernyTurner configuration with computer-optimized optics to reduce astigmatism. The spectrograph is suitably interfaced with an ultrasensitive CCD camera (PIXIS 100B). The used CCD camera is Peltier cooled and thereby enabling one to detect week Raman signal with sufficient resolution.

All the samples, except AgN, used in this study were commercially available and used without further purification. $\mathrm{AgN}$ is prepared by chemical synthesis by following the same procedures reported in [17]. A multitude of chemical reduction methods have been used to synthesize AgN from silver salts. For all the methods, some experimental conditions are rigorously controlled so as to obtain chemically stable AgN collide of specific size. The method of AgN synthesis designed for this experiment is based on the controlled reduction of silver nitrate $\left(\mathrm{AgNO}_{3}\right)$ by sodium borohydride $\left(\mathrm{NaBH}_{4}\right)$. For this purpose, commercially available $\mathrm{AgNO}_{3}$ (99.8\%) and $\mathrm{NaBH}_{4}$ (99\%) were purchased from Sigma Aldrich (Germany). Nano pure water was used as the dissolving media for both $\mathrm{AgNO}_{3}$ and $\mathrm{NaBH}_{4}$.

A $10 \mathrm{~mL}$ volume of $1.0 \mathrm{mM} \mathrm{AgNO}$, was added drop wise (roughly 1 drop per second) to $30 \mathrm{~mL}$ of $2.0 \mathrm{mM} \mathrm{NaBH}_{4}$ solution that had been chilled in an ice bath. The reaction mixture was stirred vigorously in a controlled manner on a magnetic stir plate. The solution turned a brighter yellow when all of the silver nitrate had been added. The entire addition took about three minutes, after which the stirring was stopped and the stir bar removed. The AgN colloid prepared through this protocol would have size less than $50 \mathrm{~nm}$ [17].

\section{Results and Discussion}

For the course of experiment, we have selected some hazardous organic compounds (such as benzene, aniline, chlorobenzene, and pyridine) and some typical dyes (such as R6G, Ketone Red and Prussian blue) having well characterized Raman fingerprints. Since some of the selected organic compounds (such as Ketone red, pyridine, Prussian blue, etc.) have strong absorption around UV-to-visible region, the $514.5 \mathrm{~nm}$ excitation of the Argon ion laser for the Raman measurements of these compounds resulted in strong fluorescence. Therefore, we could not measure the Raman signal for those samples using Argon-Ion laser. To avoid the inherent florescence problem, we used the Diode laser which emits 782 wavelengths, at which wavelength the selected compounds do not have appreciable absorption and thereby do not exhibit measurable fluorescence.

Figures $2(a, c)$ and $2(b, d)$ display the spectra of pyridine/Prussian blue measured with Argon-Ion laser excitation at $514.5 \mathrm{~nm}$ and Diode laser excitation at $782 \mathrm{~nm}$, respectively. It is apparent from Figures 2(a) and 2(c) that the Argon-Ion laser excitation at $514.5 \mathrm{~nm}$ gives broad, diffused, and intense fluorescence signal of both the compounds. On the other hand, Diode laser excitation at $782 \mathrm{~nm}$ yielded reasonably structured Raman fingerprints of the two compounds with almost negligible fluorescence background. Therefore, from Figure 2, one can easily assess that, albeit producing comparatively week Raman signals, excitation at $782 \mathrm{~nm}$ from Diode laser in our modified Raman system offers a suitable way of diminishing strong fluorescence signal of a compound and thus enabling to measure the corresponding Raman signals.

In addition, some of the selected compounds (such as benzene, nitrobenzene, and chlorobenzene) exhibit almost no fluorescence. For such nonfluorescent compounds, we 


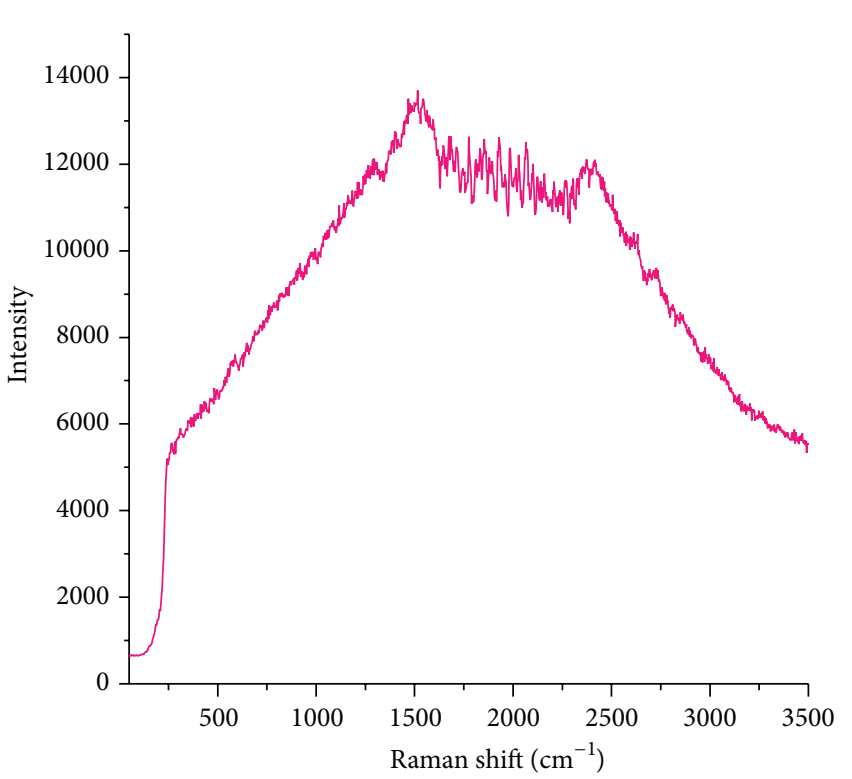

(a) Florescence of pyridine

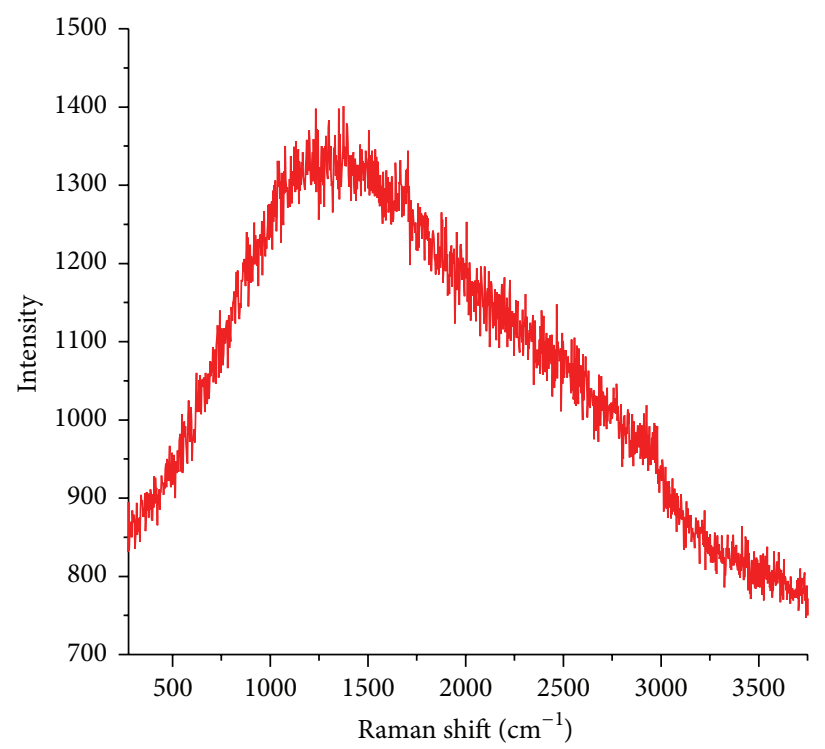

(c) Florescence of Prussian Blue

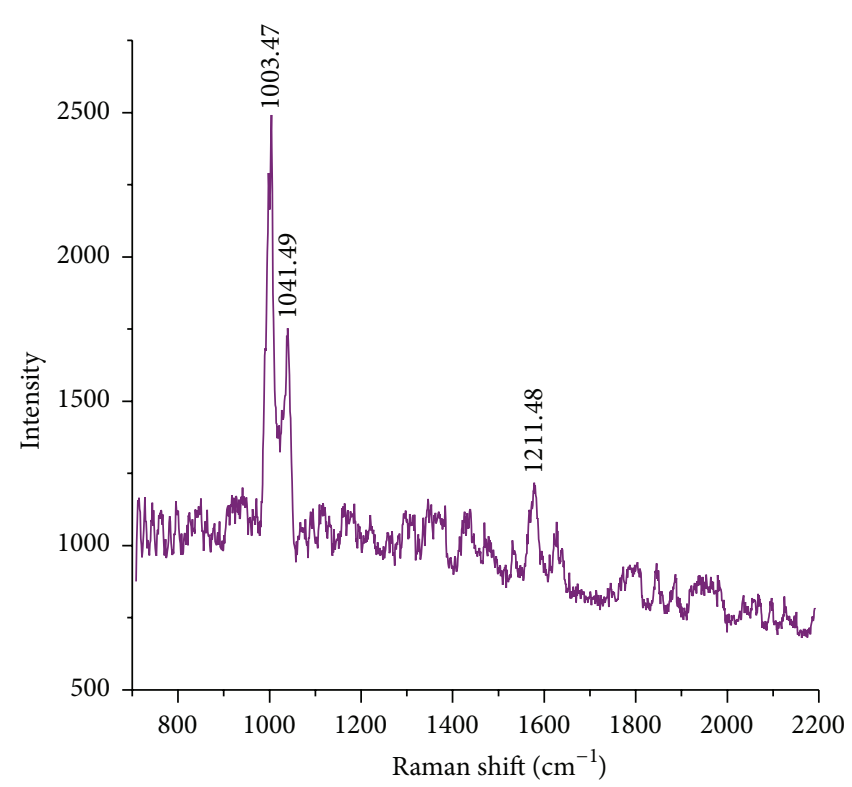

(b) Raman signal of pyridine

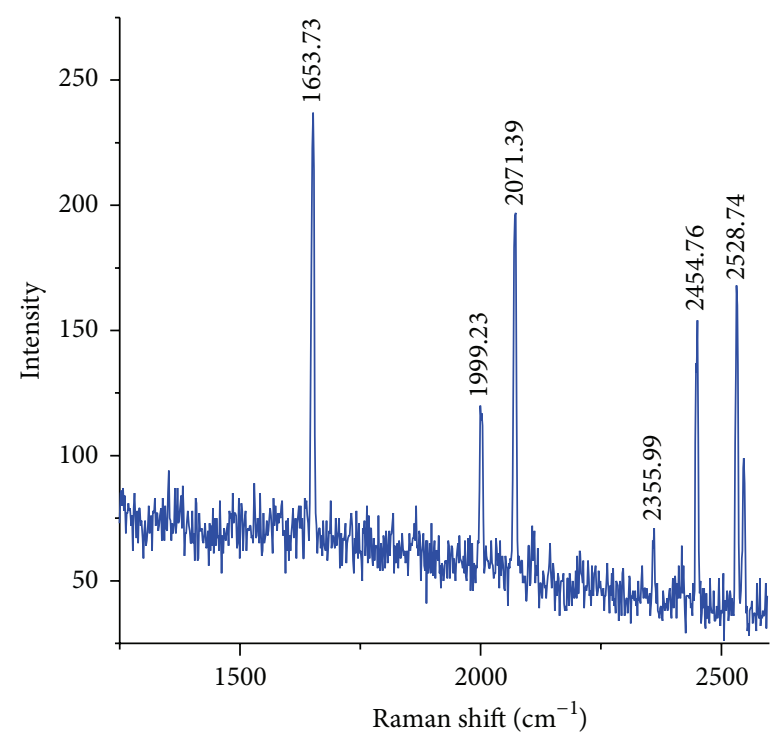

(d) Raman Signal of Prussian Blue

Figure 2: (a, b) Fluorescence/Raman signal of pyridine measured by Ar-Ion/Diode laser. (c, d) Fluorescence/Raman signal of Prussian blue measured by Argon-Ion/Diode laser.

observed notably larger Raman signal intensity with ArgonIon laser than the Diode laser, which is reasonable as the Raman intensity varies as the inverse of the fourth power of the excitation wavelength. To show the intensity variation, the Raman signals of benzene and nitrobenzene were taken using the Argon-Ion laser and also the Diode laser excitation with the same experimental condition (Figure 3).

One can easily observe from the two figures that, for the two nonfluorescent compounds with the same experimental condition, the Argon-Ion laser can yield significantly stronger Raman intensity than the Diode laser. This result in fact points the better usability of the Argon-Ion laser towards measuring Raman signals of the non-fluorescent molecules compared to the Diode laser. Similarly, we obtained much stronger Raman signal using the Argon-Ion laser than the diode laser from other nonfluorescent organic compounds such as chlorobenzene and aniline (spectra not shown). We then compiled the Raman shifts of different vibrational modes of aniline, chlorobenzene, nitrobenzene, and benzene (Table 1) and made a comparison with the values available in literature.

From Table 1, one can see that the values of Raman shifts, obtained through the Raman scattering, due to different vibrational modes of different samples lie very close (within 


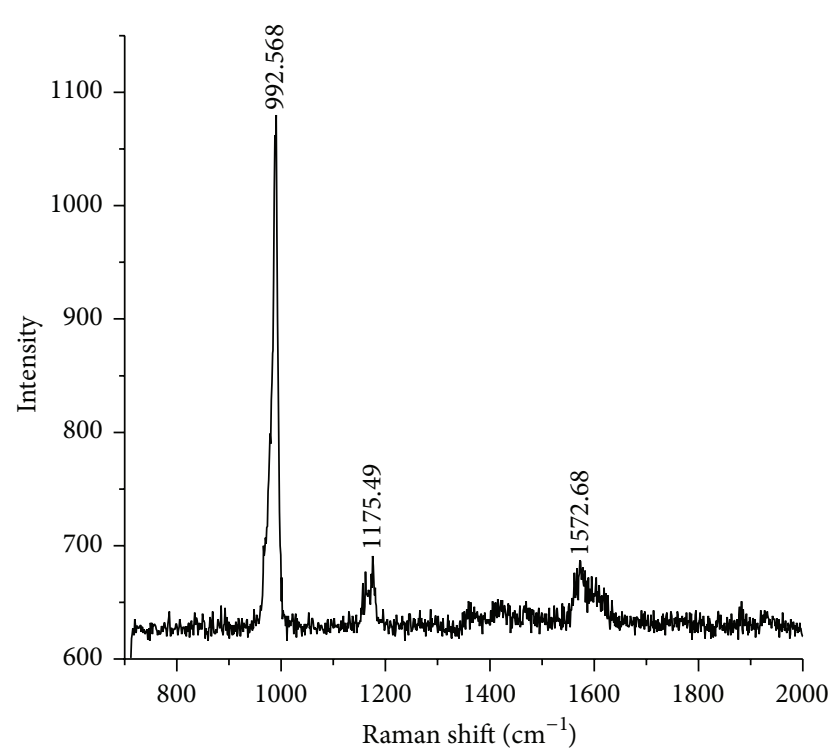

(a) Raman Signal of benzene by Diode laser

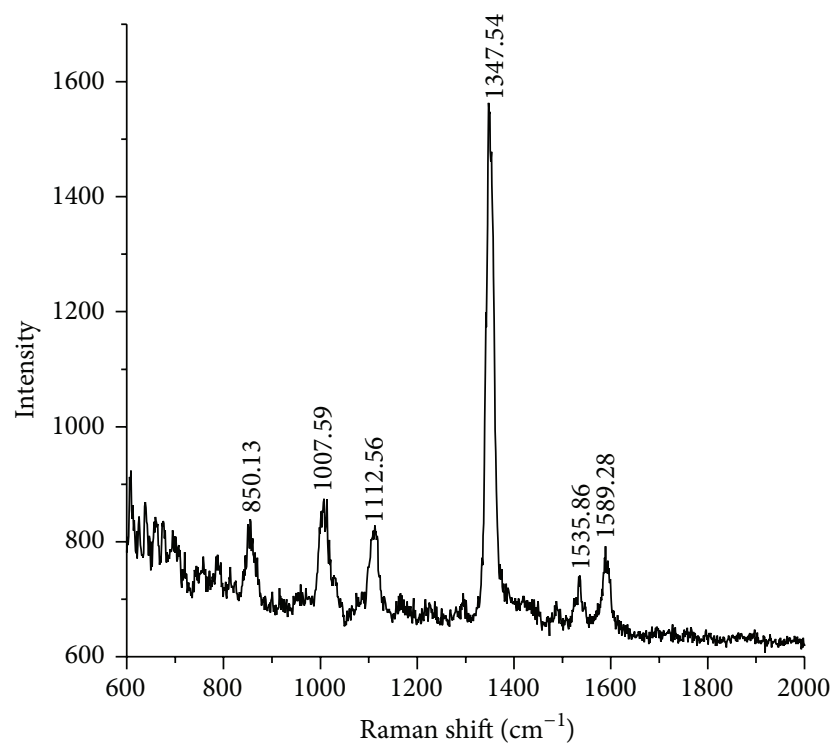

(c) Raman Signal of nitrobenzene by Diode laser

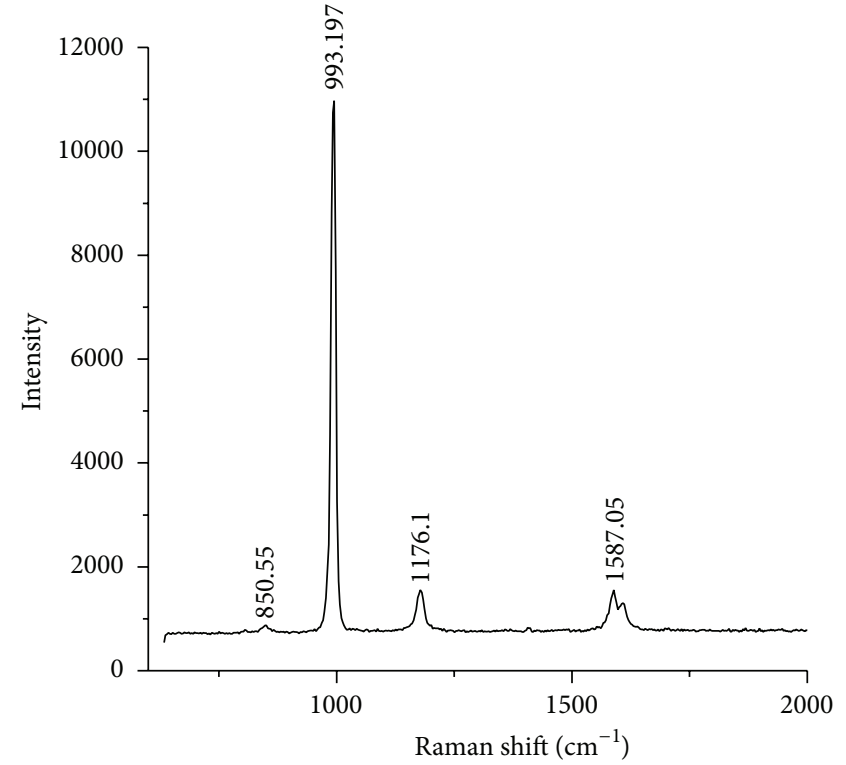

(b) Raman Signal of benzene by Argon-Ion laser

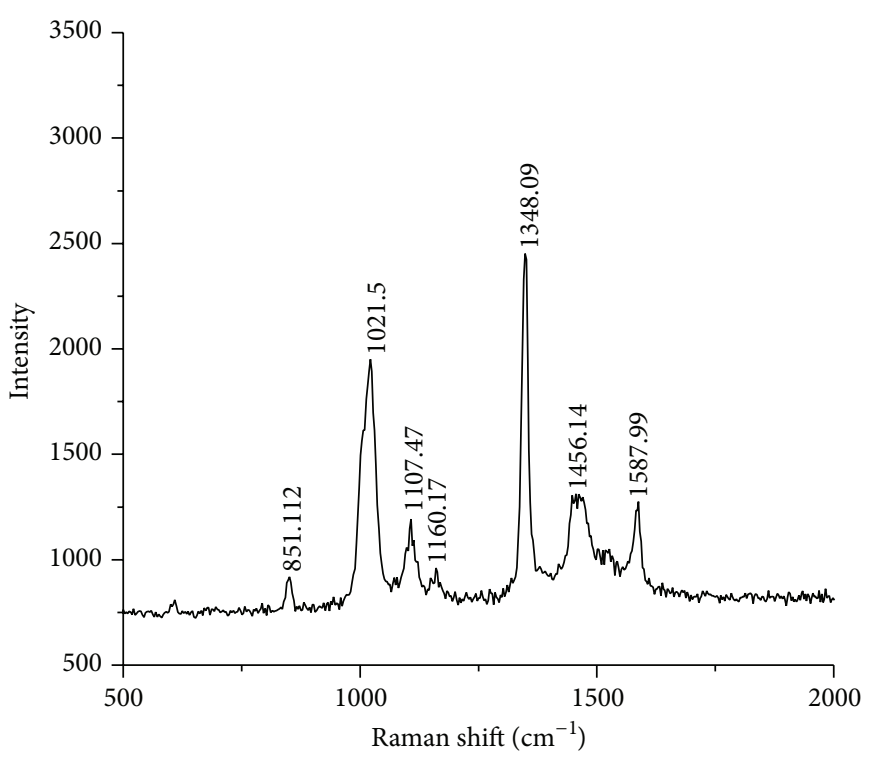

(d) Raman Signal of nitrobenzene by Argon-Ion laser

FIgUre 3: $(\mathrm{a}, \mathrm{b})$ and $(\mathrm{c}, \mathrm{d})$ depict the Raman spectra of Benzene/Nitrobenzene taken with Diode laser/Argon-Ion laser, respectively.

TABLE 1: Raman shift of benzene, aniline, chlorobenzene, and nitrobenzene with their corresponding vibrational bands and Literature values.

\begin{tabular}{|c|c|c|c|c|c|c|c|c|}
\hline \multicolumn{2}{|c|}{$\begin{array}{l}\text { Aniline } \\
\left(\mathrm{cm}^{-1}\right)\end{array}$} & \multicolumn{2}{|c|}{$\begin{array}{l}\text { Chlorobenzene } \\
\left(\mathrm{cm}^{-1}\right)\end{array}$} & \multicolumn{2}{|c|}{$\begin{array}{l}\text { Nitrobenzene } \\
\quad\left(\mathrm{cm}^{-1}\right)\end{array}$} & \multicolumn{2}{|c|}{$\begin{array}{c}\text { Benzene } \\
\left(\mathrm{cm}^{-1}\right)\end{array}$} & \multirow{2}{*}{ Band } \\
\hline $\begin{array}{l}\text { Measured } \\
\text { value }\end{array}$ & $\begin{array}{c}\text { Literature } \\
\text { value }\end{array}$ & $\begin{array}{l}\text { Measured } \\
\text { value }\end{array}$ & $\begin{array}{l}\text { Literature } \\
\text { value }\end{array}$ & $\begin{array}{l}\text { Measured } \\
\text { value }\end{array}$ & $\begin{array}{l}\text { Literature } \\
\text { value }\end{array}$ & $\begin{array}{l}\text { Measured } \\
\text { value }\end{array}$ & $\begin{array}{l}\text { Literature } \\
\text { value }\end{array}$ & \\
\hline 531.8 & 531 & & & & & & & $\omega(\mathrm{C}-\mathrm{C})$ \\
\hline 618.7 & 619.5 & 610.4 & 609 & 614.7 & & 608 & 609.17 & $\omega(\mathrm{C}-\mathrm{C})$ \\
\hline \multirow[t]{2}{*}{996.8} & 996 & 1002.7 & 1001 & & & 992 & 993.19 & $\omega(\mathrm{C}-\mathrm{C})$ \\
\hline & & & & 1021.5 & & & & $\delta(\mathrm{C}-\mathrm{H})$ \\
\hline 1150.5 & 1150 & 1156.2 & 1156 & 1160.2 & 1159 & 1178 & 1176.5 & $\delta(\mathrm{C}-\mathrm{H})$ \\
\hline 1604.4 & 1603 & 1581.9 & 1580 & 1587.9 & 1590 & 1588 & 1587.1 & $\omega(\mathrm{C}-\mathrm{C})$ \\
\hline 3057.2 & 3056 & 3068 & 3068 & 3081.1 & 3080 & 3061 & 3061.1 & $v(\mathrm{C}-\mathrm{H})$ \\
\hline
\end{tabular}




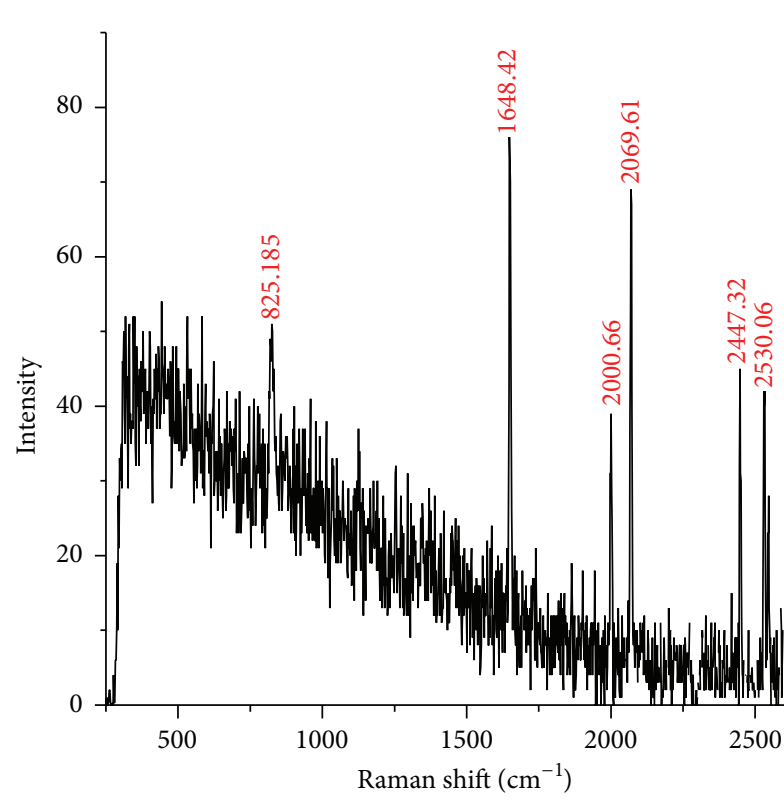

(a) Raman Signal of R6G

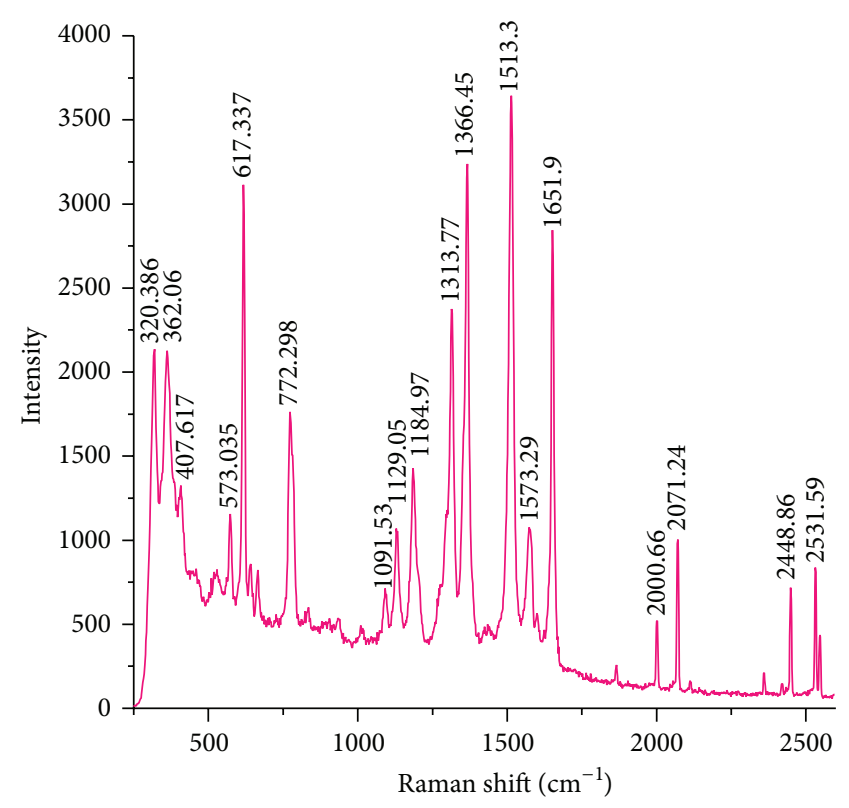

(b) Raman Signal of R6G with AgN

Figure 4: (a) Raman Signal of R6G without AgN and (b) Raman Signal of R6G with AgN with acquisition time $60 \mathrm{s.}$

TABLE 2: Comparison between literature values and experimental values of different vibrational modes of Rhodamine 6G.

\begin{tabular}{lccc}
\hline Sample & $\begin{array}{c}\text { Vibrational modes and } \\
\text { functional groups }\end{array}$ & $\begin{array}{c}\text { Literature values } \\
\left(\mathrm{cm}^{-1}\right)[12]\end{array}$ & $\begin{array}{c}\text { Experimental values } \\
\left(\mathrm{cm}^{-1}\right)\end{array}$ \\
\hline C-H op bend & 785 & 772.3 \\
C-H ip bend & & 1186.9 \\
Rhodamine 6G & Arom C-C str & 1303 & 1313.3 \\
& Arom C-C str & 1357 & 1366.66 \\
& Arom C-C str & 1508 & 1513.3 \\
\hline
\end{tabular}

$\pm 2 \mathrm{~cm}^{-1}$ ) to the values reported in literature for those bands $[18,19]$. This in fact demonstrates the reasonable accuracy and reliability of the present Raman system.

Then, we have extended our work to obtain SERS upon adsorbing the probe R6G onto the AgN prepared by chemical synthesis. Figure 4(a) shows the Raman signal of the R6G at the acquisition time $60 \mathrm{~s}$ using diode laser and Figure 4(b) shows the Raman signal of R6G with chemically prepared $\mathrm{AgN}$ with the same acquisition time and same laser. Note that R6G is strongly fluorescent with Argon-Ion laser excitation and thus we have used the Diode laser excitation for measuring its Raman spectrum.

From Table 2, one can see that the values of Raman shifts due to different vibrational modes of R6G, obtained through the SERS protocol, lie close to the values reported in literature [12]. This in fact demonstrates further the reasonable accuracy and reliability of the present Raman system.

In Figure 5, we overlaid the two Raman spectra in the same layout to see at a glance the resulting enhancement of the signals. One can easily notice that the Raman band intensities of R6G throughout the selected spectral region experience huge enhancement when measured upon incubation with the AgN collide. We have estimated the magnitude of enhancement for different Raman bands. The average enhancement of the Raman intensity was found to $10^{3}$.

In addition to the overall intensity enhancement, some more Raman features are also readily apparent especially around $0-1500 \mathrm{~cm}^{-1}$, indicating that the Raman modes of R6G residing around this region suffer larger extent of enhancement. This observation can be interpreted by considering the fact that the Raman spectrum under SERS conditions can be affected by the Plasmon resonances (producing the enhancement) which are typically wavelength dependent [20]. As a consequence, different parts of the spectrum can be amplified by different amounts, depending on the dispersion of the underlying resonance producing the enhancement.

\section{Conclusion}

In this paper, we report the use of a dispersive Laser Raman system operable with three different excitation sources (Argon-ion, He-Ne, and Diode lasers) and applied the system 


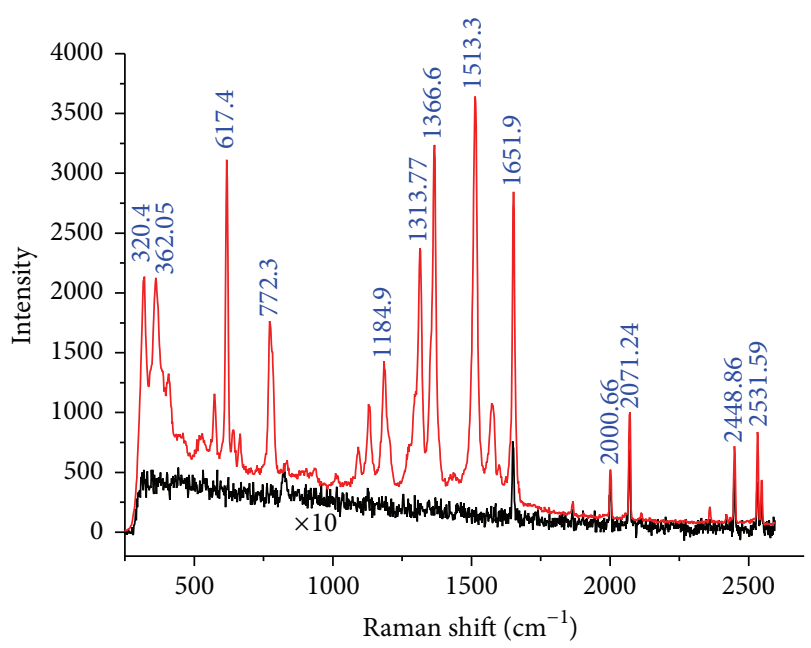

Figure 5: Raman signal of R6G with (red curve) and without (black curve) AgN.

to a variety of Raman active (hazardous organic compounds and dyes) molecules, some of which are strongly fluorescent. Then, an effort was made to evaluate the potential of Raman spectroscopy to achieve SERS activity. The chemical analysis of liquid samples containing organic components either as the main constituent or as a contaminant was of our prime interest. Raman spectra of some well-known and basic solvents were probed and verified with literature values. The spectra of the samples under investigation could be well acquired with a spectral range $200-4000 \mathrm{~cm}^{-1}$ and the shifting of prominent bands were less than $\pm 2 \mathrm{~cm}^{-1}$ which proves the accuracy of our system. We used frequently the Argon-Ion laser $(514.5 \mathrm{~nm})$ and Diode laser $(782 \mathrm{~nm})$ for the Raman excitation.

Following are the three important parts, what we obtained from our Laser Raman system. (i) We found that the accuracy of our Raman system lies within the range $\pm 2 \mathrm{~cm}^{-1}$. (ii) During our experiment, we used mostly two different excitation sources: Diode laser with wavelength at $782 \mathrm{~nm}$ and ArgonIon laser with excitation wavelength at $514.5 \mathrm{~nm}$. We observed that Argon-Ion laser is more efficient for nonfluorescent compounds. Besides, we found that Diode laser serves as very suitable source of excitation towards elimination of inherent fluorescence of some compounds under Raman study. (iii) The most exciting and important part of our experiment was to obtain SERS using the chemically synthesized $\mathrm{AgN}$, in which case the average enhancement of the Raman intensity was found to be $10^{3}$.

It is to be mentioned here that, due to the huge dumping of industrial byproducts, most of the rivers in our country are receiving a large volume of hazardous materials and composites every day. The continuous dumping of mostly untreated industrial affluent is imposing a severe threat not only to the surrounding inhabitants dependent directly or indirectly to the river water but also the underneath living bodies and aquatic microorganism and thus throwing a big challenge to the overall biodiversity in the surrounding areas. Among the other possible pollutants, hazardous organic and inorganic composites give direct effects to the water pollution, as many of them may have good dissolving strength to water. Therefore, the proper profiling of the pollutant constituents appears to be a fundamental prerequisite for the better treatment of the polluted water for the use of river bank inhabitants and to underneath aquatic microorganisms. Therefore, our effort of trace detection through SERS would mark a definite progress in the development of SERS based detection systems for different hazardous organic pollutants in our country.

\section{Conflict of Interests}

The authors declare that there is no conflict of interests regarding the publication of this paper.

\section{Acknowledgment}

The experimental work was done by using the facilities of the Nonlinear Optics and Laser Spectroscopy Laboratory of the Center for Advanced Research in Sciences (CARS) of the University of Dhaka.

\section{References}

[1] B. Schrader, Ed., Infrared and Raman Spectroscopy: Methods and Applications, John Wiley \& Sons, Chichester, UK, 1995.

[2] J. M. Chalmers and P. R. Griffiths, Handbook of Vibrational Spectroscopy, John Wiley \& Sons, Chichester, UK, 2002.

[3] J. R. Ferraro, K. Nakamoto, and C. W. Brown, Introductory Raman Spectroscopy, Academic Press, New York, NY, USA, 2003.

[4] A. Campion and P. Kambhampati, "Surface-enhanced Raman scattering," Chemical Society Reviews, vol. 27, no. 4, pp. 241-250, 1998.

[5] M. Jain and L. R. Lindvold, "Blue light stimulation and linearly modulated optically stimulated luminescence," Ancient TL, vol. 25, no. 2, pp. 69-80, 2007.

[6] R. A. Meyers, "Interpretation of infrared spectra," in Encyclopedia of Analytical Chemistry, John Wiley \& Sons, Chichester, UK, 2000.

[7] X. Wang, T. He, H. Wen, C. Xu, J. Zuo, and F. C. Liu, "Surfaceenhanced Raman scattering from citrate, azobenzene, pyridine and cyanine dyes adsorbed on $\mathrm{Ag}_{2} \mathrm{O}$ colloids," Spectrochimica Acta Part A: Molecular and Biomolecular Spectroscopy, vol. 53, no. 9, pp. 1411-1417, 1997.

[8] A. Sabur, M. Havel, and Y. Gogotsi, "SERS intensity optimization by controlling the size and shape of faceted gold nanoparticles," Journal of Raman Spectroscopy, vol. 39, no. 1, pp. 61-67, 2008.

[9] R. M. Stöckle, Y. D. Suh, V. Deckert, and R. Zenobi, "Nanoscale chemical analysis by tip-enhanced Raman spectroscopy," Chemical Physics Letters, vol. 318, no. 1-3, pp. 131-136, 2000.

[10] B. Pettinger, B. Ren, G. Picardi, R. Schuster, and G. Ertl, "Nanoscale probing of adsorbed species by tip-enhanced Raman spectroscopy," Physical Review Letters, vol. 92, Article ID 096101, 2004.

[11] E. C. Le Ru and P. G. Etchegoin, "Single-molecule surfaceenhanced raman spectroscopy," Annual Review of Physical Chemistry, vol. 63, pp. 65-87, 2012. 
[12] A. M. Michaels, M. Nirmal, and L. E. Brus, "Surface enhanced Raman spectroscopy of individual rhodamine $6 \mathrm{G}$ molecules on large Ag nanocrystals," Journal of the American Chemical Society, vol. 121, no. 43, pp. 9932-9939, 1999.

[13] K. M. Abedin, S. F. U. Farhad, M. R. Islam, M. A. I. Talukder, and A. F. M. Y. Haider, "Construction and operation of a dispersive laser Raman spectrograph using interference filter," Journal of Bangladesh Academy of Sciences, vol. 32, no. 1, pp. 114-122, 2008.

[14] K. M. Abedin, M. A. Habib, M. Wahadoszamen, A. I. Talukder, and A. F. M. Y. Haider, "Construction of laser raman system using diode laser and its performance," Journal of Bangladesh Academy of Sciences, vol. 33, no. 1, pp. 55-62, 2009.

[15] S. Farid Uddin Farhad, K. M. Abedin, M. Rafiqul Islam, A. I. Talukder, and A. F. M. Y. Haider, "Determination of ratio of unsaturated to total fatty acids in edible oils by laser Raman spectroscopy," Journal of Applied Sciences, vol. 9, no. 8, pp. 15381543, 2009.

[16] Encyclopedia of Laser Physics and Technology, vol. 2, John Wiley \& Sons, Chichester, UK, 2008.

[17] S. D. Solomon, M. Bahadory, A. V. Jeyarajasingam, S. A. Rutkowsky, C. Boritz, and L. Mulfinger, "Synthesis and study of silver nanoparticles," Journal of Chemical Education, vol. 84, no. 2, pp. 322-325, 2007.

[18] G. Varsanyi, Vibrational Spectra of Benzene Derivatives, Akademiai Kiado, Budapest, Hungary; Academic Press, New York, NY, USA, 1969.

[19] P. Kolek, K. Pirowska, Ł. Chacaga, and J. Najbar, "LIF excitation spectra of jet-cooled 3,5-dicyanoaniline," Physical Chemistry Chemical Physics, vol. 5, no. 19, pp. 4096-4107, 2003.

[20] J. M. Pitarke, V. M. Silkin, E. V. Chulkov, and P. M. Echenique, "Theory of surface plasmons and surface-plasmon polaritons," Reports on Progress in Physics, vol. 70, no. 1, pp. 1-87, 2007. 

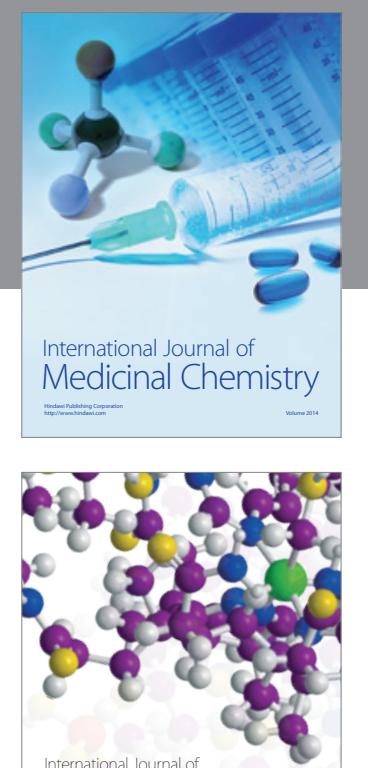

\section{Carbohydrate} Chemistry

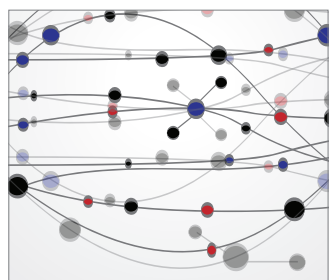

The Scientific World Journal
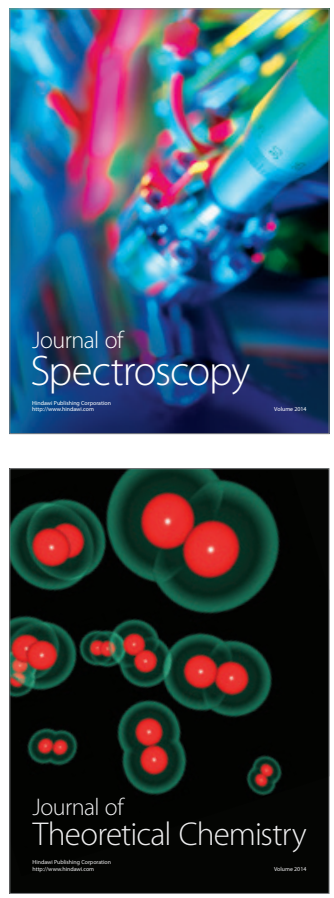
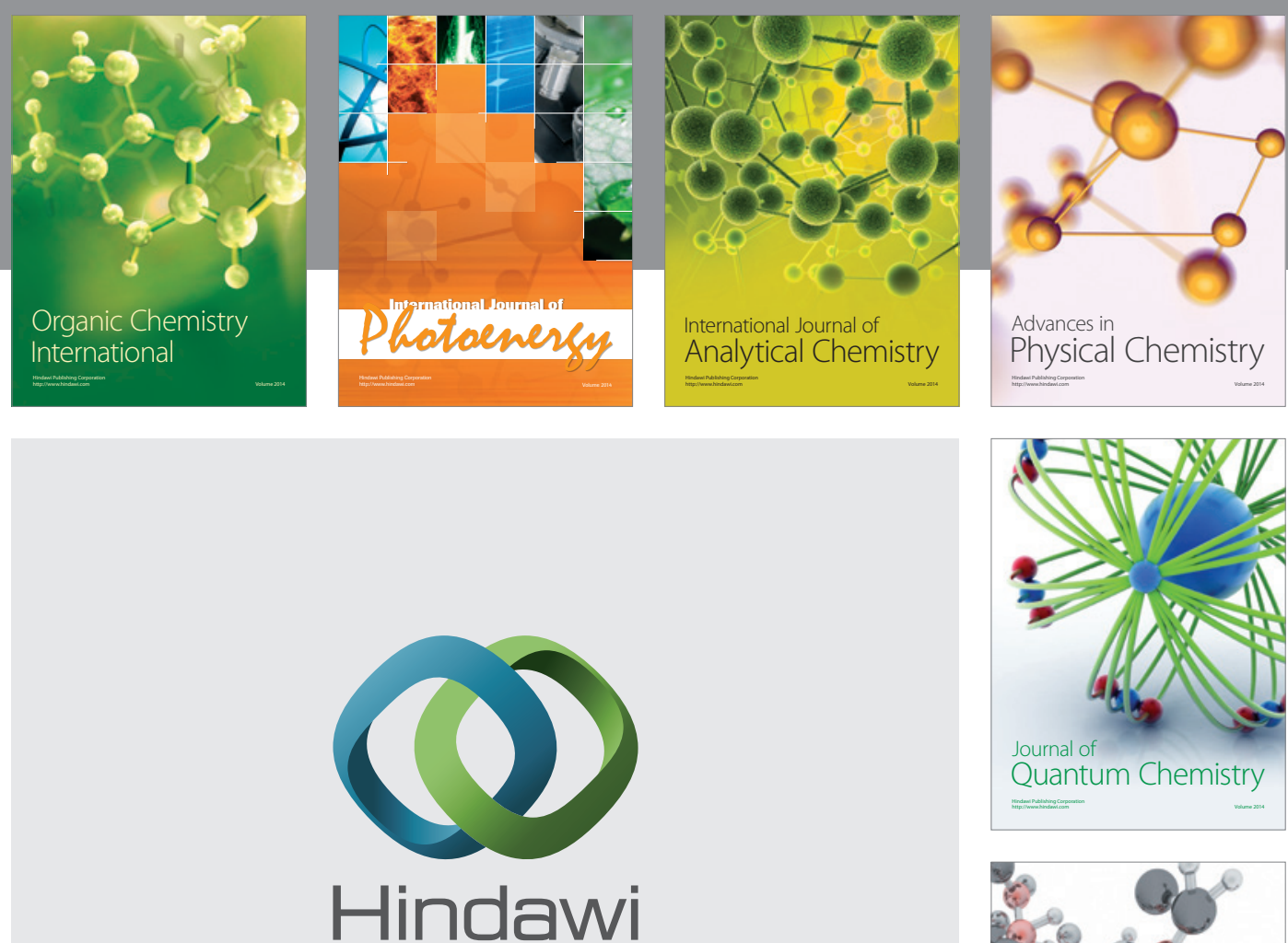

Submit your manuscripts at

http://www.hindawi.com

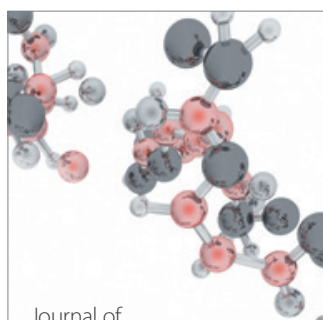

Analytical Methods

in Chemistry

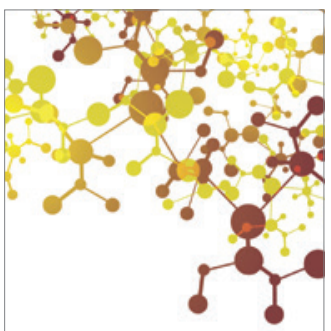

Journal of

Applied Chemistry

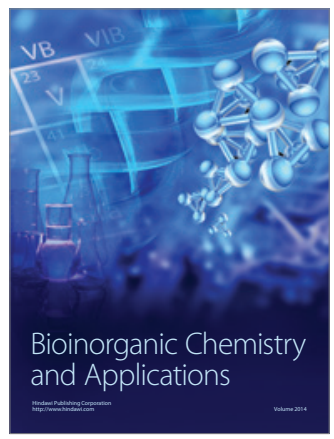

Inorganic Chemistry
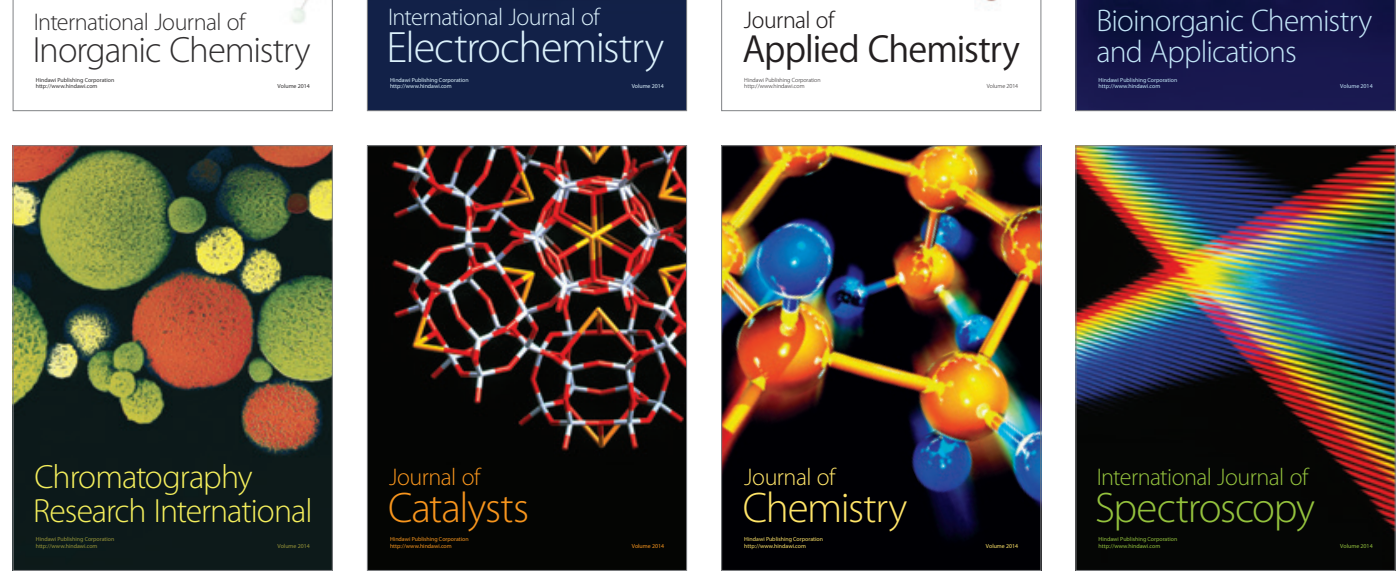